\section{Commentary: Redo cardiac surgery: Striving for the best but prepared for the worst}

\author{
Christopher Tarola, MD, FRCSC, and \\ Stephen Fremes, MD, FRCSC
}

The increased morbidity and mortality associated with reoperative cardiac surgery are well documented by singlecenter experiences. More recently, an adjusted analysis of propensity-matched cohorts by Bianco and colleagues ${ }^{1}$ demonstrated a significant increase in operative mortality $(8.37 \%$ vs $6.07 \%)$ and associated excess mortality at 30 days (hazard ratio [HR], 1.36), 1 year (HR, 1.3), and 5 years (HR, 1.3) among propensity-matched cohorts. Similar findings exist in the redo coronary bypass, valvular, and aortic surgical populations. ${ }^{2-4}$ As surgeons continue to face an increasing number of redo operations in often older patients, whether due to increased life expectancy or changes to surgical recommendations and patient preferences (ie, increasing use of bioprosthetic valves among younger patients ${ }^{5}$ ), it is critical for surgeons to have a thorough understanding of reoperative risks and outcomes.

In this issue of the Journal, Kindzelski and colleagues ${ }^{6}$ describe their outcomes in reoperative cardiac surgery with prior sternotomy among 6627 patients, excluding those undergoing heart transplant or endovascular stenting of the thoracic aorta. The investigation's focus is outcomes based, with particular attention drawn to the utility of cardiopulmonary bypass (CPB) initiation before (early) or after (late) redo sternotomy, generally on the basis of operative risk screening. The cohort was stratified into 755 versus 5872 patients who received early versus late $C P B$,

\footnotetext{
From the Division of Cardiac Surgery, Department of Surgery, Sunnybrook Health Sciences Center, Toronto, Ontario, Canada.

Disclosures: The authors reported no conflicts of interest.

The Journal policy requires editors and reviewers to disclose conflicts of interest and to decline handling or reviewing manuscripts for which they may have a conflict of interest. The editors and reviewers of this article have no conflicts of interest.

Received for publication Jan 18, 2021; revisions received Jan 18, 2021; accepted for publication Jan 20, 2021; available ahead of print Jan 28, 2021.

Address for reprints: Stephen Fremes, MD, FRCSC, Division of Cardiac Surgery, Sunnybrook Health Sciences Center, 2075 Bayview Ave, Toronto, Ontario M4N 3M5, Canada (E-mail: stephen.fremes@sunnybrook.ca).

J Thorac Cardiovasc Surg 2022;164:1767-8 0022-5223/\$36.00

Copyright (c) 2021 Published by Elsevier Inc. on behalf of The American Association for Thoracic Surgery

https://doi.org/10.1016/j.jtcvs.2021.01.068
}

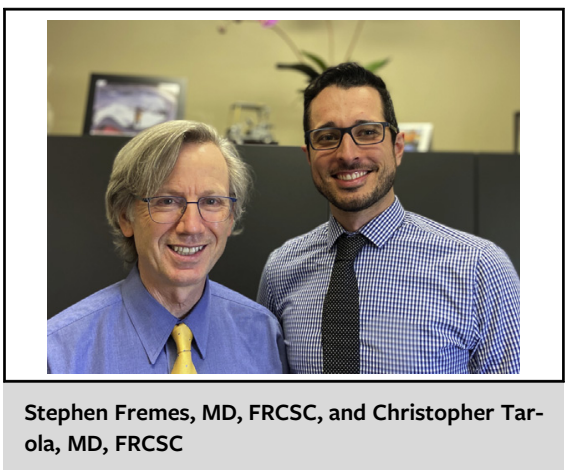

\author{
CENTRAL MESSAGE \\ Purposeful preoperative plan- \\ ning and use of the multidisci- \\ plinary heart team may help \\ reduce the surgical risks associ- \\ ated with reoperative cardiac \\ surgery to levels comparable \\ with primary surgery.
}

respectively, and each group subclassified to high versus low risk reentry based on the following criteria: sternal adherence of bypass grafts crossing midline, ascending aorta adherence to the sternum, and pseudoaneurysm in close proximity to the sternum. In both the high- and low-risk surgical groups, there was no difference in propensity-weighted operative mortality and long-term survival among patients who received an early or late $\mathrm{CPB}$ strategy. ${ }^{6}$

Foremost, the authors should be commended for their excellent outcomes, having achieved $3.5 \%$ all-comer mortality. However, this result should be interpreted in the context of this institution's experience, having completed more than 7500 redo operations in less than 10 years, or approximately 750 redo operations annually, with more than $18 \%$ re-redo operations. The conclusion that reoperation does not confer increased procedural risk is likely generalizable to large-volume, highly experienced centers. This group has improved on the $4.5 \%$ mortality reported in their 2008 cohort, ${ }^{7}$ which demonstrates the effectiveness of continuous quality improvement and evidences a potential pathway forward toward reducing surgical risk with reoperation that may be generalizable to a greater number of surgical centers.

The authors highlight the importance of implementing preoperative screening protocols to evaluate perioperative 
risk and use of the heart team model. All patients received preoperative contrast-enhanced computed tomography when possible (if not, then noncontrast) to evaluate the risk of sternal reentry and operative approach, and complex cases were reviewed by a multidisciplinary heart team. This ensures that the current range of surgical options can be explored. The heart team should maintain regularly scheduled conferences to screen complex cases for operative risk, and it is important that decisions are based not only on literature-based evidence but also on patient choices and institutional experience.

Recent cohort studies from several jurisdictions have suggested that valve-in-valve is a lower risk strategy than redo aortic valve replacement for failing bioprostheses, at least for moderate-risk patients. $^{8-10}$ Kindzelski and colleagues ${ }^{6}$ instead maintain that prior sternotomy in and of itself should not be an automatic indication to defer to a more minimally invasive surgical approach. Rather, multidisciplinary heart teams should comprehensively risk stratify patients to optimize outcomes, and decisions should consider local expertise.

Although the choice of instituting early versus late CPB likely can be left to the operating surgeon on a case-by-case, risk-stratified basis, Kindzelski and colleagues ${ }^{6}$ highlight the importance of preoperative planning and preparedness while encouraging the cardiac surgical community that redo surgery can consistently result in a positive outcomes and may trend toward outcomes similar to primary surgery.

\section{References}

1. Bianco V, Kilic A, Gleason TG, Aranda-Michel E, Habertheuer A, Wang Y, et al. Reoperative cardiac surgery is a risk factor for long-term mortality. Ann Thorac Surg. 2020;110:1235-42

2. Launcelott S, Ouzounian M, Buth KJ, Legare JF. Predicting in-hospital mortality after redo cardiac operations: development of a preoperative scorecard. Ann Thorac Surg. 2012;94:778-84.

3. Yap CH, Sposato L, Akowuah E, Theodore S, Dinh DT, Shardey GC, et al. Contemporary results show repeat coronary artery bypass grafting remains a risk factor for operative mortality. Ann Thorac Surg. 2009;87: 1386-91.

4. Onorati F, Perotti A, Reichart D, Mariscalco G, Ratta ED, Santarpino G, et al. Surgical factors and complications affecting hospital outcome in redo mitral surgery: insights from a multicentre experience. Eur J Cardiothorac Surg. 2016;49: $127-33$.

5. Isaacs A, Shuhaiber J, Salemi A, Isom OW, Sedrakyan A. National trends in utilization and in-hospital outcomes of mechanical versus bioprosthetic aortic valve replacements. J Thorac Cardiovasc Surg. 2015;149:1262-9.

6. Kindzelski BA, Bakaeen FG, Tong MZ, Roselli EE, Soltesz EG, Johnston DR, et al. Modern practice and outcomes of reoperative cardiac surgery. J Thorac Cardiovasc Surg. 2022;164:1755-66.e16.

7. Roselli EE, Pettersson GB, Blackstone EH, Brizzio ME, Houghtaling PL, Hauck R, et al. Adverse events during reoperative cardiac surgery: frequency, characterization, and rescue. J Thorac Cardiovasc Surg. 2008;135: 316-23.

8. Tam DY, Dharma C, Rocha RV, Ouzounian M, Wijeysundera HC, Austin PC, et al. Transcatheter ViV versus redo surgical AVR for the management of failed biological prosthesis: early and late outcomes in a propensity-matched cohort. JACC Cardiovasc Interv. 2020;13:765-74.

9. Hirji SA, Percy ED, Zogg CK, Malarczyk A, Harloff MT, Yazdchi F, et al. Comparison of in-hospital outcomes and readmissions for valve-in-valve transcatheter aortic valve replacement vs reoperative surgical aortic valve replacement: a contemporary assessment of real-world outcomes. Eur Heart J. 2020;41: 2747-55.

10. Deharo P, Bisson A, Herbert J, Lacour T, Etienne CS, Porto A, et al. Transcatheter valve-in-valve aortic valve replacement as an alternative to surgical re-replacement. J Am Coll Cardiol. 2020;76:489-99. 